\title{
MULTI-OBJECTIVE OPTIMISATION OF THE ELECTRIC WHEELCHAIR RIDE COMFORT AND ROAD HOLDING BASED ON JOURDAIN'S PRINCIPLE MODEL AND GENETIC ALGORITHM
}

\author{
Mohamed BELHORMA ${ }^{* \oplus,}$, Aboubakar Seddik BOUCHIKHI ${ }^{*}$ \\ *Faculty of Technology; Department of Mechanical Engineering, Mechanics of Structures and Solids Laboratory, \\ University of Sidi Bel Abbes, BP 89, Cité Ben Mhidi, Sidi Bel Abbes 22000, Algeria \\ medbelhorma@hotmail.com, asbouchikhi@yahoo.fr
}

received 23 March 2021, revised 15 November 2021, accepted 30 November 2021

\begin{abstract}
The paper addresses the multi-body modelling of an electric wheelchair using Jourdain's principle. First, a description of the adopted approach was presented. Next, the mathematical equations were developed to obtain the dynamic behaviour of the concerned system. The numerical computation was performed with MATLAB (matrix laboratory: a high performance language of technical computing) and validated by MBD (Multi-Body Dynamics) for Ansys, a professional multi-body dynamics simulation software powered by RecurDyn. Afterwards, the model was treated as an objective function included in genetic algorithm. The goal was to improve the ride quality and the road holding as well as the suspension workspace. The multi-objective optimisation aimed to reduce the Root-Mean-Square (RMS) of the seat's vertical acceleration, the wheels load and the workspace modulus by varying the bodies' masses, the spring-damper coefficients and the characteristics of the tires. Acceptable solutions were captured on the Pareto fronts, in contrast to the relatively considerable processing time involved in the use of a random road profile generated by the power spectral density (PSD). During the process, the compatibility and the efficiency of Jourdain's equations were inspected.
\end{abstract}

Key words: multi-body systems, Jourdain's principle, electric wheelchair, multi-objective optimisation, genetic algorithm

\section{INTRODUCTION}

Recent systems are complex and consist of many bodies interconnected by joints and elements of force. These systems are called multi-body systems in the literature. The dynamic equations that generate the motion of these systems are highly non-linear and thus in most cases cannot be solved by a closed analytical form, making the numerical solution of the resulting equations indispensable. Therefore, a proper choice of the formalism of multi-body modelling can improve numerical efficiency.

In 1909, Jourdain [16] had an intention to find a bridge between d'Alembert's principle and the Gauss principle of least constraint, and he did so by publishing a paper about a third principle of mechanics. This generalised form of d'Alembert's principle uses virtual velocities instead of virtual displacements, and is applied to systems with differential nonholonomic constraints [27]. Papastavridis [22] has demonstrated that Jourdain's principle results naturally from the total time differentiation of Lagrange's principle and produces the correct equations of motion independently of any commutation assumptions. Inexplicably, this principle seems to be very little known, and also appears prone to be confused with Kane's equations [17] presented in 1961 as "a general method for obtaining the differential equations of nonhoIonomic systems". Some authors state that Kane's equations are merely a reformulation of the concepts Appell and Jourdain developed earlier, due to which Piedboeuf [24] has reviewed both Jourdain's principle and Kane's equations and demonstrated that the latter can be expressed as easily the former. Henceforth,
Jourdain's principle will be applied in modelling dynamical behaviour of mechanical systems such as a powered wheelchair.

Electric wheelchairs are helpful for people unable to use a manual wheelchair, especially for larger distances or over rough terrain. In case a wheelchair is provided with a suspension, as shown in the example of Fig. 1, it will have an increased performance.

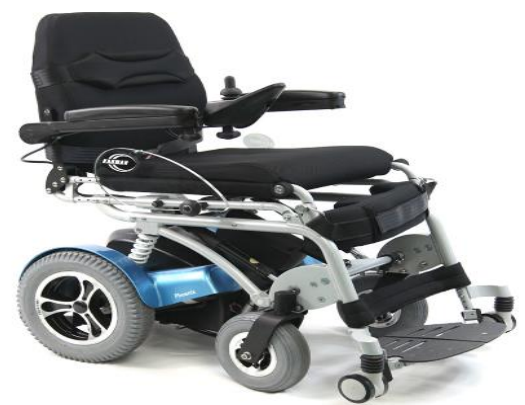

Fig. 1. The KARMAN XO-202 electric wheelchair [12]

To improve its performance, an electric-powered wheelchair may be subject to different types of studies. Vingback et al. [34] have used a motion base simulator to facilitate fast and costefficient development and adjustment of wheel suspension systems and parameters for increasing the ride comfort of wheelchair-seated passengers. Wang et al. [35] have compared the vibration isolation performances of the convalescent-wheelchair robot with and without a vibration-reducing device, and conducted 
the sensitivity analysis of the vibration responses to the important dynamic parameters. Although there exist many works dealing with the development of active and semi-active suspension systems, such as the researches of Van der Sande et al. [33], Anandan and Kandavel [2] and others [4, 8, 20, 29, 31] on multiobjective optimisation related to passenger comfort and road holding of ground vehicles, few dealt with multi-objective optimisation of electric wheelchairs. The focus was on improving control automatisms using genetic algorithms, in contrast with the studies of Sankardoss and Geethanjali [28], Ahmad et al. [1] and Dad et al. [5]. However, there is a lack of powered wheelchair design optimisation that combines evolutionary algorithms and Jourdain's equations.

This article will provide a clear description of the multi-body modelling of an electric wheelchair according to Jourdain's principle, from the generation of equations of motion to the numerical computation. Of particular interest are the interaction between the first-order ordinary differential equations (ODEs) and the nonlinearity of the forces applied by the suspension as well as the random road effect produced by the power spectral density (PSD). The validated model will be injected into an optimisation program in order to investigate its efficiency, robustness and rapidity as a function with multiple objectives. The choice will be on the genetic algorithm in the MATLAB Optimisation Toolbox to improve ride quality and road holding. The multi-objective optimisation will aim to reduce the Root-Mean-Square (RMS) of the seat's vertical acceleration, the tires load and the suspension workspace modulus by varying the bodies' masses, the spring-damper coefficients and some parameters of the tires. The dominant objectives will be extracted, and the influence of the normalised variables will be examined. The reader will notice the frequent use of the predefined functions of MATLAB, both in modelling and optimisation, the aim of which is to benefit from a combination between these tools and first-order differential equations by having a straightforward process.

\section{JOURDAIN'S PRINCIPLE OF VIRTUAL POWER}

According to Rill [26], a right-handed Cartesian coordinate system is fixed to the body in its centre of mass (CoG: Centre of Gravity). The position and the orientation of body $i$ with respect to the inertial reference frame 0 (RF0) is determined by the position vector $r$ and the rotation matrix $A$, respectively.

$r_{0 i, 0}=r_{0 i, 0}(y)$

and

$$
A_{0 i, 0}=A_{0 i, 0}(y)
$$

where the generalised coordinates $y_{1}, y_{2}, \ldots, y_{n}$ are collected in the vector $y$. The velocity of body $i$ in RF0 is

$$
v_{0 i, 0}=\frac{d}{d t} r_{0 i, 0}(y)=\sum_{m=1}^{f} \frac{\partial r_{0 i, 0}(y)}{\partial y_{m}} \dot{y}_{m}=v_{0 i, 0}(y, \dot{y})
$$

The time derivative of $A_{0 i} . A_{0 i}^{T}$ is a skew-symmetric matrix

$$
\widetilde{\omega}_{0 i, 0}=\left[\begin{array}{ccc}
0 & -\omega_{0 i, 0}(3) & \omega_{0 i, 0}(2) \\
\omega_{0 i, 0}(3) & 0 & -\omega_{0 i, 0}(1) \\
-\omega_{0 i, 0}(2) & \omega_{0 i, 0}(1) & 0
\end{array}\right]
$$

where the vector of angular velocity

$$
\omega_{0 i, 0}=\left[\omega_{0 i, 0}(1), \omega_{0 i, 0}(2), \omega_{0 i, 0}(3)\right]^{T}
$$

For simplification, we replace by $z$, where $z=K(y) \dot{y}$, $z=z(y, \dot{y}), v_{0 i, 0}(y, z)$ and $\omega_{0 i, 0}(y, z)$. The time derivative of velocities yields to

$a_{0 i, 0}=\sum_{m=1}^{f} \frac{\partial v_{0 i, 0}(y, z)}{\partial y_{m}} \dot{y}_{m}+\sum_{m=1}^{f} \frac{\partial v_{0 i, 0}(y, z)}{\partial z_{m}} \dot{z}_{m}$

$\alpha_{0 i, 0}=\sum_{m=1}^{f} \frac{\partial \omega_{0 i, 0}(y, z)}{\partial y_{m}} \dot{y}_{m}+\sum_{m=1}^{f} \frac{\partial \omega_{0 i, 0}(y, z)}{\partial z_{m}} \dot{z}_{m}$

The motion of one rigid body is described by Newton-Euler equations

$$
\begin{aligned}
& m_{i} \cdot a_{0 i, 0}=F_{i, 0} \\
& \Theta_{i, 0} \cdot \alpha_{0 i, 0}+\omega_{0 i, 0} \times \Theta_{i, 0} \cdot \omega_{0 i, 0}=T_{i, 0}
\end{aligned}
$$

where $m_{i}$ is the mass of body $i$ and $\Theta_{i, 0}$ is the corresponding inertia tensor. The forces and torques for constrained systems are devoted to ones applied on body $i$ and others provided by constraints

$F_{i, 0}=F_{i, 0}^{a}+F_{i, 0}^{c}$

$T_{i, 0}=T_{i, 0}^{a}+T_{i, 0}^{c}$

The partial velocities and partial angular velocities are arranged in the $3 \times f$ Jacobian matrices of translation and rotation, as under:

$\frac{\partial v_{0 i, 0}}{\partial z}=\left[\frac{\partial v_{0 i, 0}(y, z)}{\partial z_{1}}, \frac{\partial v_{0 i, 0}(y, z)}{\partial z_{2}}, \ldots, \frac{\partial v_{0 i, 0}(y, z)}{\partial z_{f}}\right]$

$\frac{\partial \omega_{0 i, 0}}{\partial z}=\left[\frac{\partial \omega_{0 i, 0}(y, z)}{\partial z_{1}}, \frac{\partial \omega_{0 i, 0}(y, z)}{\partial z_{2}}, \ldots, \frac{\partial \omega_{0 i, 0}(y, z)}{\partial z_{f}}\right]$

Using the Jacobian matrices, the accelerations are obtained as:

$a_{0 i, 0}=\frac{\partial v_{0 i, 0}}{\partial z} \dot{z}+a_{0 i, 0}^{R}$

$\alpha_{0 i, 0}=\frac{\partial \omega_{0 i, 0}}{\partial z} \dot{z}+\alpha_{0 i, 0}^{R}$

The remaining terms in the accelerations are presented by $R$. It should be mentioned that the reaction terms are vanished. The first-order differential equation is $M(y) \dot{z}=q(y, z)$, where $f \times f$ mass matrix is defined by

$M(y)=\sum_{i=1}^{k}\left[\frac{\partial v_{0 i, 0}^{T}}{\partial z} m_{i} \frac{\partial v_{0 i, 0}}{\partial z}+\frac{\partial \omega_{0 i, 0}^{T}}{\partial z} \Theta_{i, 0} \frac{\partial \omega_{0 i, 0}}{\partial z}\right]$

and the $f \times 1$ vector of generalised forces is given by

$q(y, z)=\sum_{i=1}^{k}\left[\frac{\partial v_{0 i, 0}^{T}}{\partial z} H_{1}+\frac{\partial \omega_{0 i, 0}^{T}}{\partial z} H_{2}\right]$

where

$H_{1}=F_{i, 0}^{a}-m_{i} \cdot a_{0 i, 0}^{R}$

and

$H_{2}=T_{i, 0}^{a}-\Theta_{i, 0} \cdot \alpha_{0 i, 0}^{R}-\omega_{0 i, 0} \times \Theta_{i, 0} \cdot \omega_{0 i, 0}$

\section{DYNAMICAL MODEL OF THE ELECTRIC WHEELCHAIR}

\subsection{Kinematics}

The Non-Linear Two-Dimensional Electric Wheelchair (NL2DEWC), presented in Fig. 2, consists of four rigid bodies: Chassis 
or Sprung mass (S), Control arm (C), Front Tire (FT) and Rear Tire (RT). The motion of the system is described by four generalised coordinates $\mathrm{y}=\left(\begin{array}{llll}z & \gamma & \beta & \varphi\end{array}\right)^{T}$ representing the vertical displacement of the Sprung mass, the rotation angle of the Control arm about the revolute joint $\mathrm{Bc}$, the rotation angle of the $\mathrm{FT}$ about the revolute joint $\mathrm{Bf}$ and the rotation angle of the RT about the revolute joint $\mathrm{Br}$, respectively. The tires' deflections rsf and rsr, and the step inputs uf and ur of the road, are illustrated in Fig. 2.

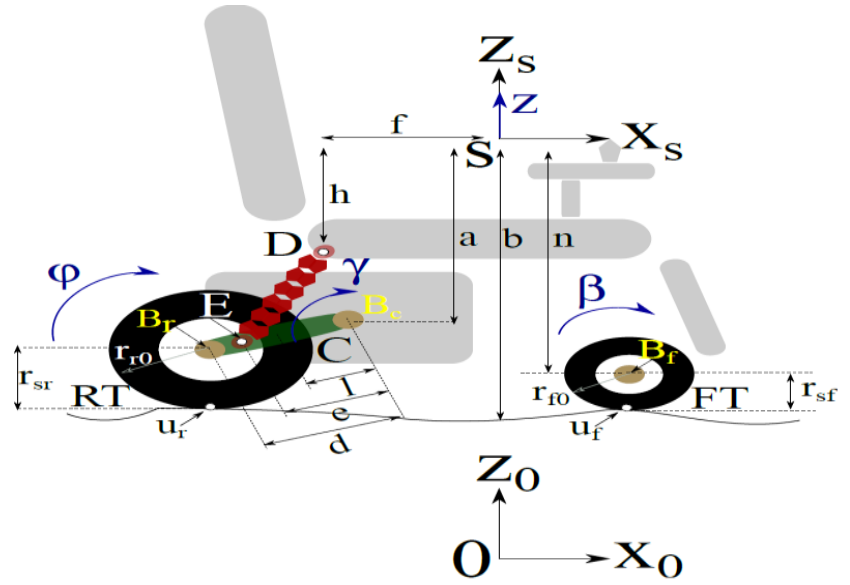

Fig. 2. The NL2D-EWC. FT, front tire; NL2D-EWC, non-linear two-dimensional electric wheelchair; RT, rear tire

The position, velocity and acceleration of the Sprung mass' CoG are

$p_{s}=\left(\begin{array}{c}0 \\ 0 \\ b+z\end{array}\right), v_{s}=\left(\begin{array}{l}0 \\ 0 \\ \dot{z}\end{array}\right), a_{s}=\left(\begin{array}{l}0 \\ 0 \\ \ddot{z}\end{array}\right)$

The distance $b$ between the Sprung mass' COG and the ground, and the parameters $I, d, a, n, r_{r}, r_{f 0}, h, f, d_{2}$ and $e$ are shown in Fig. 2. The NL2D-EWC is not considered as a pitchplane model because there is no orientation for the Sprung mass. Therefore, the rotation matrix that describes the orientation of the Control arm with respect to the Sprung mass' Reference Frame (SRF) is the same that describes it in RF0.

${ }_{s}^{c} A={ }_{0}^{c} A=\left[\begin{array}{ccc}\cos \gamma & 0 & \sin \gamma \\ 0 & 1 & 0 \\ -\sin \gamma & 0 & \cos \gamma\end{array}\right]$

and the angular velocity and angular acceleration are

$\omega_{c}=\left(\begin{array}{c}0 \\ \dot{\gamma} \\ 0\end{array}\right)$, and $\alpha_{c}=\left(\begin{array}{c}0 \\ \ddot{\gamma} \\ 0\end{array}\right)$

The position vector is

$p_{c}=\left(\begin{array}{c}0 \\ 0 \\ b+z\end{array}\right)+\left(\begin{array}{c}0 \\ 0 \\ -a\end{array}\right)+\left(\begin{array}{c}-l \cos \gamma \\ 0 \\ l \sin \gamma\end{array}\right)$

The first two parts characterise the position of the revolute joint $\mathrm{B}_{\mathrm{c}}$. The first and second derivatives yield to

$v_{c}=\left(\begin{array}{c}0 \\ 0 \\ \dot{z}\end{array}\right)+\left(\begin{array}{c}l \dot{\gamma} \sin \gamma \\ 0 \\ l \dot{\gamma} \cos \gamma\end{array}\right)$

and the acceleration $a_{c}=\left(\begin{array}{c}0 \\ 0 \\ \ddot{z}\end{array}\right)+\left(\begin{array}{c}l \ddot{\gamma} \sin \gamma \\ 0 \\ l \ddot{\gamma} \cos \gamma\end{array}\right)+\left(\begin{array}{ccc}l & \dot{\gamma}^{2} & \cos \gamma \\ & 0 \\ -l & \dot{\gamma}^{2} \sin \gamma\end{array}\right)$

The orientation of the FT with respect to the RF0 is determined by

${ }_{0}^{F T} A=\left[\begin{array}{ccc}\cos \beta & 0 & \sin \beta \\ 0 & 1 & 0 \\ -\sin \beta & 0 & \cos \beta\end{array}\right]$

and the momentary position is determined by

$p_{F T}=\left(\begin{array}{c}0 \\ 0 \\ b+z-n\end{array}\right)$

then the velocity and acceleration are given by

$\omega_{F T}=\left(\begin{array}{c}0 \\ \dot{\beta} \\ 0\end{array}\right), \alpha_{F T}=\left(\begin{array}{c}0 \\ \ddot{\beta} \\ 0\end{array}\right)$

and

$v_{F T}=\left(\begin{array}{l}0 \\ 0 \\ \dot{Z}\end{array}\right), a_{F T}=\left(\begin{array}{l}0 \\ 0 \\ \ddot{Z}\end{array}\right)$

for the RT

${ }_{0}^{R T} A=\left[\begin{array}{ccc}\cos \varphi & 0 & \sin \varphi \\ 0 & 1 & 0 \\ -\sin \varphi & 0 & \cos \varphi\end{array}\right]$

$p_{R T}=\left(\begin{array}{c}0 \\ 0 \\ b+z-a\end{array}\right)+\left(\begin{array}{cc}-d & \cos \gamma \\ 0 \\ d & \sin \gamma\end{array}\right)$

The velocity and acceleration are

$\omega_{R T}=\left(\begin{array}{c}0 \\ \dot{\varphi} \\ 0\end{array}\right), \alpha_{R T}=\left(\begin{array}{c}0 \\ \ddot{\varphi} \\ 0\end{array}\right)$

and

$v_{R T}=\left(\begin{array}{l}0 \\ 0 \\ \dot{z}\end{array}\right)+\left(\begin{array}{ccc}d & \dot{\gamma} & \sin \gamma \\ & 0 \\ d & \dot{\gamma} & \cos \gamma\end{array}\right)$

$a_{R T}=\left(\begin{array}{l}0 \\ 0 \\ \ddot{z}\end{array}\right)+\left(\begin{array}{ccc}d & \ddot{\gamma} & \sin \gamma \\ 0 & \\ d & \ddot{\gamma} & \cos \gamma\end{array}\right)+\left(\begin{array}{ccc}d & \dot{\gamma}^{2} & \cos \gamma \\ 0 & \\ -d & \dot{\gamma}^{2} & \sin \gamma\end{array}\right)$

According to Eqs (6) and (7) from the Jacobians, the partial velocities can be extracted from corresponding generalised velocities.

\subsection{Applied forces}

The complete force vector is populated from each body's gravitational forces and translational forces of the spring-damper unit; finally, the tires' longitudinal and vertical forces as it resumed is presented in Fig. 3 and Tab. 1. Translational springs are force elements in rigid-body systems. The force $F_{S}$ of a non-linear spring is defined by Hahn [11] as

$F_{S}=K_{S} \cdot \frac{r_{D E, 0}}{\left|r_{D E, 0}\right|} \cdot\left(\left|r_{D E, 0}\right|-l_{0}\right)$ 
with a positive constant $K_{S}$ as the stiffness coefficient, $l_{0}$ as the undeformed length of the spring and $r_{D E, 0}$ as the vector of the attachment points of the spring on the Sprung mass and the Control arm, the modulus of which is its deformed length. The force $F_{S}$ of the spring acts on both bodies in opposite directions where $\operatorname{sign}\left(F_{S}\right)$ on the Sprung mass $=-\operatorname{sign}\left(F_{S}\right)$ on the Control arm:

- For $\left|r_{D E, 0}\right|-l_{0}>0$ the spring is under tension and the two bodies are pulled.

- For $\left|r_{D E, 0}\right|-l_{0}<0$ the spring is under compression and the two bodies are pushed.

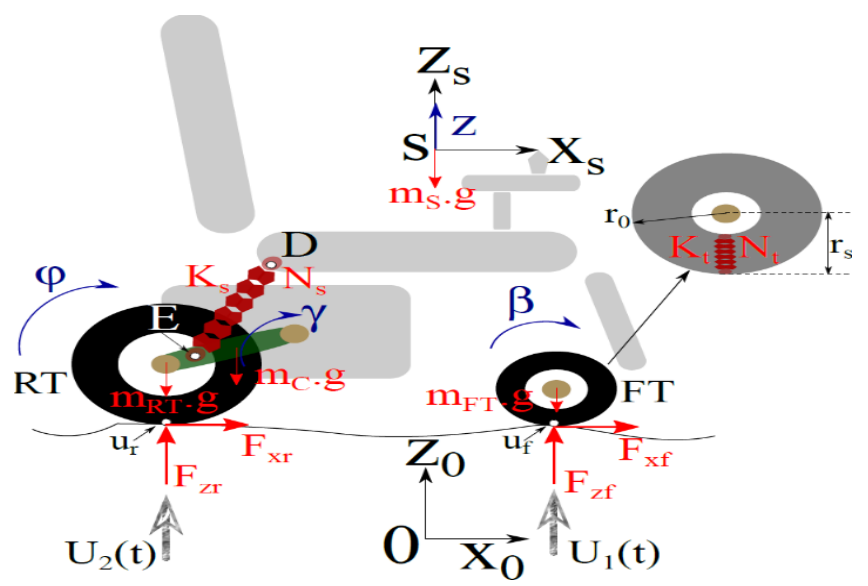

Fig. 3. Contact pressure distribution: a) gap distance effect;

b) gap orientation effect Forces acting on the NL2D-EWC. FT, front tire; NL2D-EWC, non-linear two-dimensional electric wheelchair; RT, rear tire.

For the translational damper, Hahn [11] defines the force $F_{D}$ as

$F_{D}=N_{S} \cdot \frac{r_{D E, 0}}{\left|r_{D E, 0}\right|} \cdot\left|\dot{r}_{D E, 0}\right|$

while $N_{S}$ is the suspension damping, $\dot{r}_{D E, 0}$ represents the first derivative of the deformable length, and the sign convention is as follow:

- For $\dot{r}_{D E, 0}>0$ the damper forces exhibit a pull on the bodies.

- For $\dot{r}_{D E, 0}<0$ the two bodies move towards each other, and the damper forces exhibit a push on the bodies.

Finally, the primary force of the spring-damper unit can be found as

$F_{S D}=F_{S}+F_{D}$

According to Pacejka and Besselink [21], the normal load $F_{z}$ of the wheel can be calculated as a linear function of the radial tire deflection measured by the reduction of the tire radius from the unloaded value $r_{0}$ to the loaded static radius $r_{s}$. As long as the tires are in contact with the actuator in $u_{k}(k:=$ front, rear $)$, the vertical tire force is

$F_{z k}=-c_{z k} \cdot\left(r_{0 k}-r_{s k}\right), \quad k:=$ front, rear

where

$$
\begin{aligned}
& r_{s f}=b+z-n-U_{1} \\
& r_{s r}=b+z-a+d \cdot \sin \gamma-U_{2}
\end{aligned}
$$

The constant $c_{z}$ characterises the compliance of the tires in the vertical direction. $U_{1}(t)$ and $U_{2}(t)$ are the inputs of the actuator in the points $u_{k}$ at a certain time $t_{\text {step }}$. In the following pro- cesses, the simulation of the road's profile will take the three forms shown in Fig. 4.

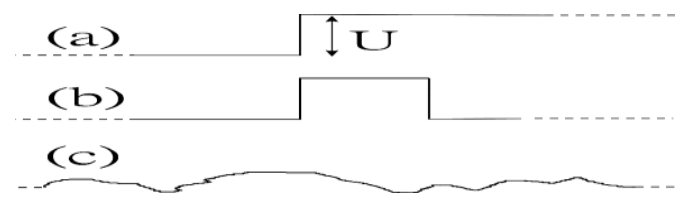

Fig. 4. Road's profiles: (a) Step, (b) Bump, (c) Random road

In the presence of adhesion in the contact area, Rill [26] defines the longitudinal tire force as

$F_{x f}=-c_{x f} \cdot\left(r_{s f} \cdot \beta\right)-d_{x f} \cdot\left(r_{s f} \cdot \dot{\beta}\right)$

$F_{x r}=-c_{x r} \cdot\left(d \cdot(1-\cos \gamma)-r_{s r} \cdot \varphi\right)-d_{x r} \cdot(d \cdot \sin \gamma \cdot \dot{\gamma}-$

$\left.r_{s r} \cdot \dot{\varphi}\right)$

The constants $c_{x k}$ and $d_{x k}(k:=$ front, rear $)$ model the tires' longitudinal compliance and damping. It must be noted that for the rear wheel, the suspension damping affects the translational motions, and the rotation is determined by the longitudinal tire force only.

\subsection{Equations of motion}

Jourdain's principle is resumed in two first-order ODEs.

$M=$

$\left[\begin{array}{cccc}m_{s}+m_{c}+m_{F T} & \left(l \cdot m_{c}+d \cdot m_{R T}\right) \cos \gamma & 0 & 0 \\ \left(l . m_{c}+d \cdot m_{R T}\right) & \Theta_{c}+l^{2} \cdot m_{c}+d^{2} \cdot m_{R T} & 0 & 0 \\ 0 & 0 & \Theta_{R T} & 0 \\ 0 & 0 & 0 & \Theta_{F T}\end{array}\right]$

$q=$
$\left(\begin{array}{c}F_{z r}+F_{z f}-m \cdot g+\left(l \cdot m_{c}+d \cdot m_{R T}\right) \cdot \sin \gamma \cdot \dot{\gamma} \\ F_{S D}-\left(l . m_{c}+d \cdot m_{R T}\right) \cdot \operatorname{los} \gamma \cdot g+d \cdot\left(F_{x r} \cdot \sin \gamma+F_{z r} \cdot \cos \gamma\right) \\ -r_{s r} \cdot F_{x r} \\ -r_{s f} \cdot F_{x f}\end{array}\right)$

where

$m=\left(m_{s}+m_{c}+m_{R T}+m_{F T}\right)$

\subsection{Numerical computation}

While the analytical techniques of Newton, D'Alembert, Lagrange and Jourdain were developed centuries ago, these classical approaches have proven to be suitable for implementation on high-speed digital computers when used with matrix and numerical methods. The application of those methods results in a group of differential equations that may be expressed in a very matrix form and might be solved using numerical and computer methods [30]. As proved previously, two ODEs sum up Jourdain's principle and they read as

$\dot{y}=x$

$M \cdot \dot{x}=q$

Kane and Levinson [18] have concluded that the process proposed here may be expected to lead to computational algorithms involving fewer arithmetic operations than algorithms generated by employing the best available Lagrangian and Newton-Euler 
approaches. The MATLAB function ode45 solves the ODEs in Eqs (43) and (44). To recover the accelerations, after the integration process is completed, knowing the coordinates and velocities at every reported time step, we recomputed the accelerations by reconstructing the equations of motion. The results will be validated next.

\section{COMPARATIVE ANALYSIS OF THE MODEL}

\subsection{Data setting}

The parameters, the geometric constants and the initial values are collected in Tabs 1, 2 and 3 in order to perform the NL2DEWC model.

Tab. 1. Model's parameters

\begin{tabular}{|c|c|c|}
\hline Designation, (symbol) & Value & Unit \\
\hline $\begin{array}{c}\text { Sprung mass with/without user's weight, } \\
\left(m_{S}\right)\end{array}$ & $130 / 50$ & $\mathrm{~kg}$ \\
\hline Mass of the control arm, $\left(m_{C}\right)$ & 2 & $\mathrm{~kg}$ \\
\hline Mass of the rear wheel, $\left(m_{R T}\right)$ & 8 & $\mathrm{~kg}$ \\
\hline Mass of the front wheel, $\left(m_{F T}\right)$ & 4 & $\mathrm{~kg}$ \\
\hline Inertia of the control arm, $\left(\Theta_{c}\right)$ & 0.6 & $\mathrm{~kg} \cdot \mathrm{m} 2$ \\
\hline Inertia of the rear wheel, $\left(\Theta_{R T}\right)$ & 0.5 & $\mathrm{~kg} \cdot \mathrm{m} 2$ \\
\hline Inertia of the front wheel, $\left(\Theta_{F T}\right)$ & 0.5 & $\mathrm{~kg} \cdot \mathrm{m} 2$ \\
\hline Suspension stiffness, $\left(K_{S}\right)$ & 10,000 & $\mathrm{~N} / \mathrm{m}$ \\
\hline Suspension damping, $\left(N_{S}\right)$ & 2,150 & $\mathrm{~N} . \mathrm{s} / \mathrm{m}$ \\
\hline Longitudinal RT stiffness, $\left(c_{x r}\right)$ & 20,0000 & $\mathrm{~N} / \mathrm{m}$ \\
\hline Longitudinal RT damping, $\left(d_{x r}\right)$ & 1,500 & $\mathrm{~N} . \mathrm{s} / \mathrm{m}$ \\
\hline Vertical RT stiffness, $\left(c_{z r}\right)$ & 98,700 & $\mathrm{~N} / \mathrm{m}$ \\
\hline Longitudinal FT stiffness, $\left(c_{x f}\right)$ & 200,000 & $\mathrm{~N} / \mathrm{m}$ \\
\hline Longitudinal front tire damping, $\left(d_{x f}\right)$ & 1,500 & $\mathrm{~N} . \mathrm{s} / \mathrm{m}$ \\
\hline Vertical front tire stiffness, $\left(c_{z f}\right)$ & 98,700 & $\mathrm{~N} / \mathrm{m}$ \\
\hline Gravity, $(g)$ & 9.81 & $\mathrm{~m} / \mathrm{s} 2$ \\
\hline
\end{tabular}

FT, front tire; RT, rear tire.

Tab. 2. Constants of the NL2D-EWC

\begin{tabular}{|c|c|}
\hline Designation,(symbol) & Value (m) \\
\hline $\begin{array}{l}\text { Distance between the joint } \mathrm{Bc} \text { and the Control arm } \\
\qquad \mathrm{CoG}, l\end{array}$ & 0.210 \\
\hline Distance between joint $\mathrm{Bc}$ and joint $\mathrm{Br}, d$ & 0.266 \\
\hline Distance between Sprung mass CoG and joint Bc, $a$ & 0.383 \\
\hline $\begin{array}{l}\text { Distance between Sprung mass } \mathrm{CoG} \text { and the ground, } \\
\qquad b\end{array}$ & 0.574 \\
\hline Distance between Sprung mass CoG and joint Bf, $n$ & 0.489 \\
\hline $\mathrm{RT}$ radius, $r r_{0}$ & 0.173 \\
\hline FT radius, $r f_{0}$ & 0.085 \\
\hline Vertical position of spring attachment $\mathrm{D}, h$ & 0.087 \\
\hline Longitudinal position of spring attachment $\mathrm{D}, f$ & 1.37 \\
\hline Distance between Front Tire centre and joint Bc, $d_{2}$ & 0.3 \\
\hline Distance between joint $\mathrm{Bc}$ and spring attachment $\mathrm{E}, e$ & 0.28 \\
\hline
\end{tabular}

FT, front tire; RT, rear tire; NL2D-EWC, non-linear two-dimensional electric wheelchair
Tab. 3. Initial values

\begin{tabular}{|c|c|c|}
\hline Designation & Value & Unit \\
\hline$z_{0}$ & 0 & $\mathrm{~m}$ \\
\hline$\gamma_{0}$ & 0 & $\mathrm{rad}$ \\
\hline$\phi_{0}$ & 0 & $\mathrm{rad}$ \\
\hline$\beta_{0}$ & 0 & $\mathrm{rad}$ \\
\hline$\dot{z}_{0}$ & 0 & $\mathrm{~m} / \mathrm{s}$ \\
\hline$\dot{\gamma}_{0}$ & 0 & $\mathrm{rad} / \mathrm{s}$ \\
\hline$\dot{\phi}_{0}$ & 0 & $\mathrm{rad} / \mathrm{s}$ \\
\hline$\dot{\beta}_{0}$ & 0 & $\mathrm{rad} / \mathrm{s}$ \\
\hline
\end{tabular}

\subsection{Model's implementation in MBD for Ansys/RecurDyn}

To validate the mathematical model developed in MATLAB, a Three-Dimensional Electric Wheelchair (3D-EWC) was built in MBD for Ansys, a professional multi-body dynamics simulation software powered by RecurDyn. The 3D-EWC appearing in Fig. 5(a) was inspired from the KARMAN's XO-202 electric wheelchair, but some differences exist concerning the design, the measurements and the objective. The seat of the model is not supposed to stand up as the KARMAN's does.

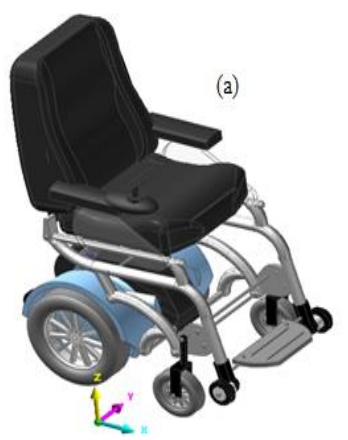

(b)

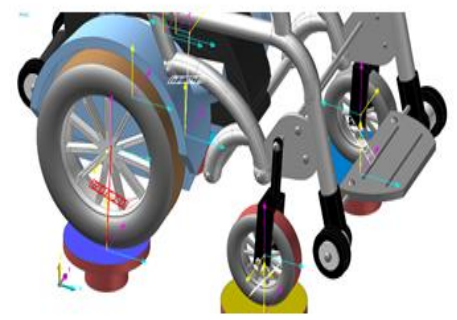

Fig. 5. (a) The 3D-EWC, (b) Tire/Actuator contact: Test-rig. 3D-EWC, three-dimensional electric wheelchair

For the designations shown in the previous tables, the NL2DEWC and the 3D-EWC have the same values but it is clear that the 3D model has a larger geometry. When the tire-road interaction is simulated by either a bumped road or a test-rig, as shown in Fig. 5(b), an adequate contact between the surfaces needs to be adjusted. Tab. 4 presents the "Solid" contact properties.

Tab. 4. Solid contact properties

\begin{tabular}{|c|c|}
\hline Designation & Value \\
\hline Bounding buffer length & $10^{9}$ \\
\hline Plane tolerance factor & 3 \\
\hline Contact spring coefficient & 3,000 \\
\hline Contact damping coefficient & 8,200 \\
\hline Rebound damping factor & 0.25 \\
\hline
\end{tabular}




\subsection{Simulation and results}

In this section, two scenarios will be presented. First, the NL2D-EWC will be treated as a quarter-car model by applying a step motion to the RT, the same as in Fig. 4(a). In $3 \mathrm{~s}$ of time simulation, the dynamical behaviour is approved by the 3D-EWC with a step that is $0.05 \mathrm{~m}$ high at $1.5 \mathrm{~s}$ and without considering the user's weight $\left(m_{S}=50 \mathrm{~kg}\right)$, as illustrated in Fig. 6 .

The figure shows the vertical displacement, velocity and acceleration of the Sprung mass in the first part where a good correlation between the modelled and simulated behaviour is noticed. In the second part of Fig. 6, the rotations of the Control arm and those of the RT are quite identical to the simulation.
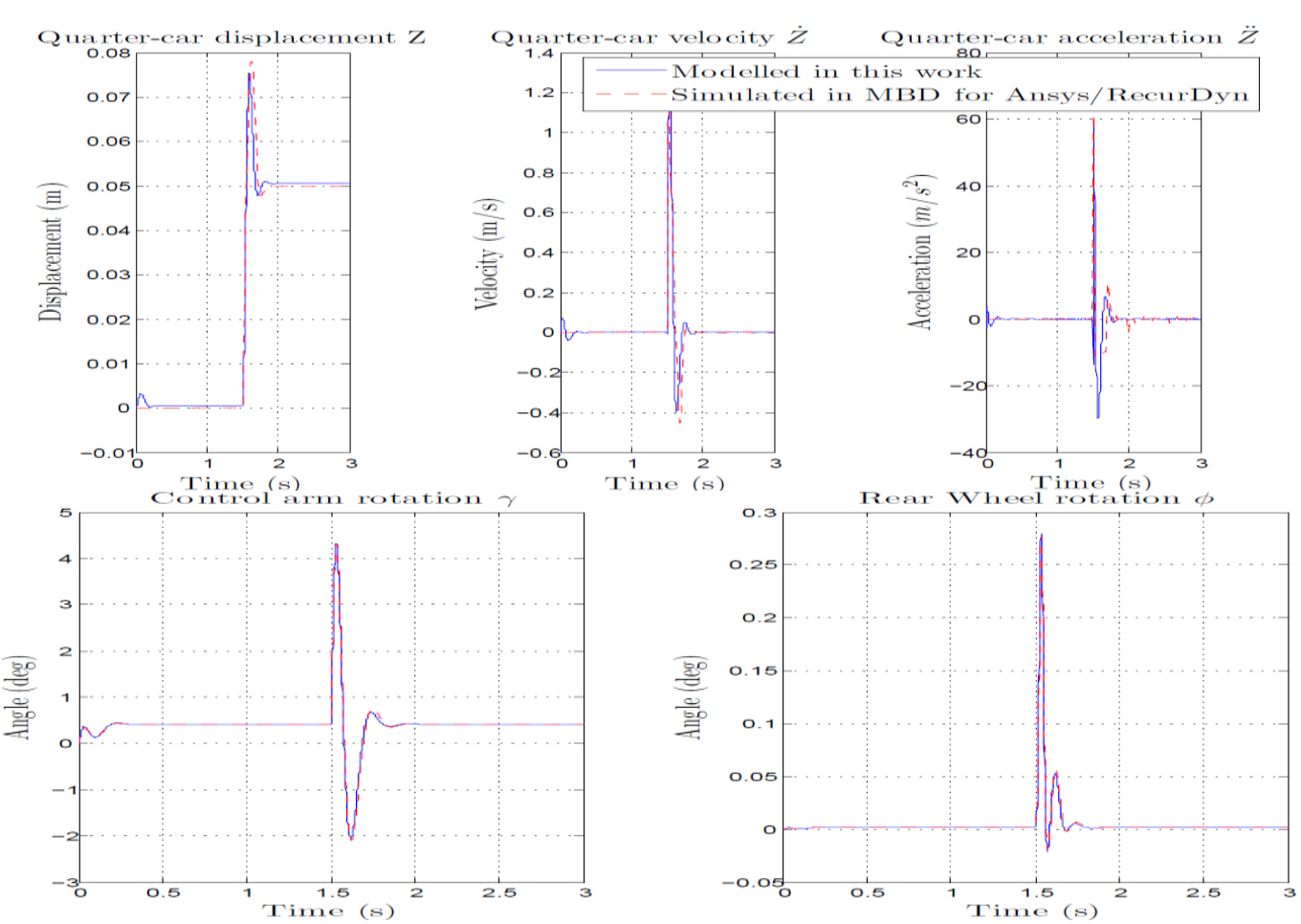

Fig. 6. NL2D-EWC quarter-car vs. MBD for Ansys model: The step case behaviour. NL2D-EWC, non-linear two-dimensional electric wheelchair

Second, the entire NL2D-EWC will be engaged by simulating a bump obstacle, as presented in Fig. 4(b), of $0.03 \mathrm{~m}$ length and $U_{1,2}=0.02 \mathrm{~m}$ high acting at two-time steps $0.25 \mathrm{~s}$ and $0.75 \mathrm{~s}$ on the FTs and RTs, respectively. The user's weight is considered and the total mass corresponding to the Sprung mass is $m_{S}=130 \mathrm{~kg}$. The NL2D-EWC gives information about the position, velocity and acceleration of the four coordinates $\mathrm{y}=$ $\left(\begin{array}{llll}z & \gamma & \beta & \varphi\end{array}\right)^{T}$. From this point, we will be interested in the vertical motion of the Sprung mass shown in Fig. 7. The bump disturbance for the front wheel is given in MBD for Ansys/RecurDyn by the following composition of functions:

STEP(time, 1.0, 0.0, 1.1, 0.02)

$$
+ \text { STEP(time, } 1.4,0.02,1.5,0.0
$$

The highest peaks in Fig. 7 are related to the displacement and acceleration of the Sprung mass when the FT passes over the obstacle, while the lower peaks are associated with the RT passage; these observations assume relevance in light of the fact that a suspension exists that connects the tire to the Sprung mass. In those experiments, there is a good agreement between the results of the mathematical and the simulated models. The small differences may be due to the linearisation of the equations of motion as it was estimated in Hurel et al. [14] or merely to the complexity of the $3 \mathrm{D}$ model over the non-linear two-dimensional one.
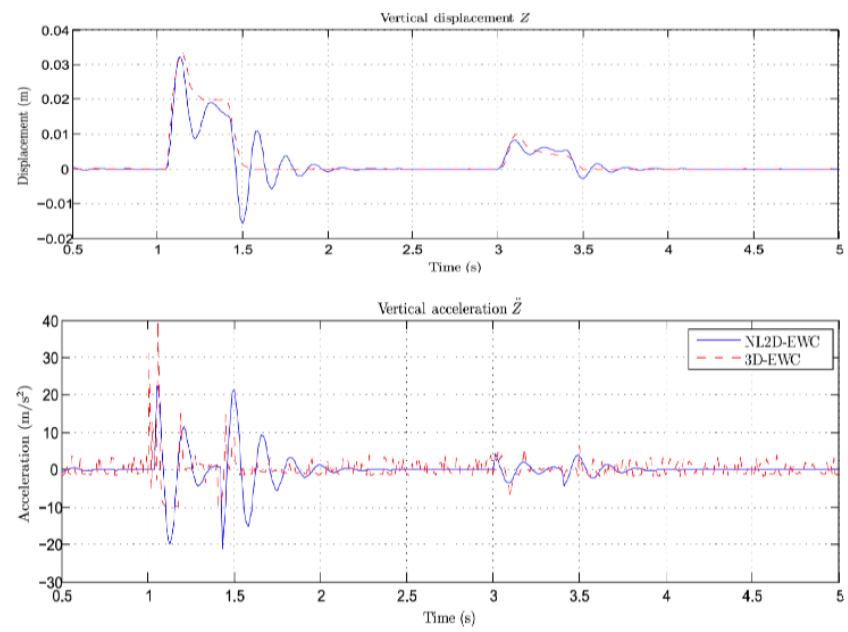

Fig.7. NL2D-EWC vs. 3D-EWC measurements: The bump case. 3D-EWC, three-dimensional electric wheelchair; NL2D-EWC, non-linear two-dimensional electric wheelchair

\section{OPTIMISATION PROCESS FOR RIDE COMFORT, ROAD HOLDING AND SUSPENSION WORKSPACE}

The optimal design of a multi-body system is started by defining an objective function, which contains the performance criteria. An objective function based on a process of multi-body dynamics 
occurs in an interval of time. It is often considered as a time integral of a function that has a set of conditions within that span [9]. For that purpose, The NL2D-EWC model based on Jourdain's principle will be included in a global optimisation program.

\subsection{Parameters of the dynamic optimisation}

The weighted RMS of the Sprung mass' acceleration is frequently used to evaluate the riding quality of a vehicle [15]. The rider's comfort improves as the acceleration decreases. The MATLAB function rms returns the RMS level of the vector $\ddot{Z}_{S}$ where the waveform is a continuous function of time [23]

$f_{1}=r m s(\ddot{Z})=\sqrt{\left(\frac{1}{t_{E n d}-t_{0}}\right) \cdot \int_{t_{0}}^{t_{E n d}} \ddot{Z}_{S}{ }^{2}(t) \cdot d t}$

The continual contact between the tires and the road surface is one of the essential factors underlying the designing of the suspension parameters. Therefore, the road holding will be a second objective. While applying the random processes theory for road holding evaluation, the RMS value of the dynamic vertical force $F_{z i}(i=$ front, rear $)$ between wheels and road, caused by the road irregularities, has been taken as the criterion for road holding. Sinha [32] concludes that for good road holding this value should have a minimum rate. The loads for FTs and RTs in Eq. (38) will be combined as

$f_{2}=\sqrt{\kappa_{f}^{2} \cdot r m s\left(F_{z f}\right)^{2}+\kappa_{r}^{2} \cdot r m s\left(F_{z r}\right)^{2}}$

$\kappa_{f}=0.6$ and $\kappa_{r}=0.4$ are coefficients imposed to regulate the combination. Due to the restriction of the structure and the vehicle's design, the space between the wheels and the suspended mass must be limited to one stroke, which is frequently referred to as the working space. To provide a better workspace, it is necessary to reduce this change in distance [29]. Consequently, the modulus of the vector between the attachment points $D$ and $E$ is chosen as the third cost function, which is given by

$f_{3}=\left\|\frac{r_{D E, 0}}{\left|r_{D E, 0}\right|} \cdot\left(\left|r_{D E, 0}\right|-l_{0}\right)\right\|$

Eq. (50) was presented previously as the deformed length of the non-linear spring-damper unit. The eight design variables are

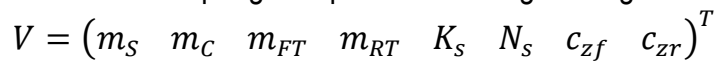

Thus, the dynamic optimisation problem can be described as

Minimise $\left\{\begin{array}{l}f_{1}(V) \\ f_{2}(V) \\ f_{3}(V)\end{array}\right.$

While the initial state of the NL2D-EWC was presented previously, the bounds of the optimisation variables are resumed in Tab. 5.

If an objective function has sensitivity to varying parameters, it may have difficulty during the process. The problem arising from comparing one value of mass and another of stiffness is expected to be clearly apparent. The transformation of the initial representation, which contains information about the physical nature, into a scaled representation is numerically efficient [10]. Each variable $V_{i}$ will be scaled to 1 by

$\widehat{V}_{i}=\frac{V_{i}-l b_{i}}{u b_{i}-l b_{i}}$ where $\widehat{V}_{i}, l b_{i}$ and $u b_{i}$ are the new scaled variable, the lower bound and the upper bound, respectively. It is necessary that the original design variables be used to calculate the objective function.

Tab. 5. Variables bonds of the dynamic optimisation

\begin{tabular}{|c|c|c|}
\hline \multirow{2}{*}{ Variables } & \multicolumn{2}{|c|}{ Bonds } \\
\cline { 2 - 3 } & lower & upper \\
\hline$m_{S}$ & 46 & 80 \\
\hline$m_{C}$ & 0.9 & 3.5 \\
\hline$m_{F T}$ & 2.5 & 4.5 \\
\hline$m_{R T}$ & 5 & 14 \\
\hline$K_{S}$ & 10,000 & 20,000 \\
\hline$N_{S}$ & 1,000 & 2,500 \\
\hline$c_{z f}$ & 90,000 & 100,000 \\
\hline$c_{z r}$ & 90,000 & 100,000 \\
\hline
\end{tabular}

\subsection{Generation of an artificial-random road profile}

The random road profile in Fig. 4(c) is chosen to be the form of excitation in the process. The literature is rich in characterisation of the road profile where most of it is made by the PSD. Doods and Robson [7] illustrate the profile classifications presented in ISO-8608 (International Organization for Standardization), from smooth irregularities (class A) to rough terrain (class E). Rill [26] and Balkwill [3] both describe an efficient generation of a random profile, which can have the form of

$\Phi(\Omega)=\Phi\left(\Omega_{0}\right) \cdot\left(\frac{\Omega}{\Omega_{0}}\right)^{-\omega}$

where the wave number expressed in $(\mathrm{rad} / \mathrm{m})$ is

$\Omega=2 \pi / L$

and the value of PSD at $\Omega_{0}=1,\left(\mathrm{~m}^{2} /(\mathrm{rad} / \mathrm{m})\right)$ is

$\Phi_{0}=\Phi\left(\Omega_{0}\right)$

$L$ represents the wavelength $(m), \Omega_{0}$ is the reference wave number $(\mathrm{rad} / \mathrm{m})$ and $\omega$ is the waviness. A sinusoidal approximation of a random profile $U_{R}$ by a superposition of $N$ $(0 \leq N \leq \infty)$ waves is

$U_{R}(s)=\sum_{i=1}^{N} A_{i} \cdot \sin \left(\Omega_{i} . s-\Psi_{i}\right)$

The momentary position of the wheelchair with the associated longitudinal speed $v_{x}$ and the phase angles $\Psi_{i}$ is given by

$s=v_{x} . t$

Eq. (56) can offer a PSD $\Phi\left(\Omega_{0}\right)$ if it has a set of amplitudes

$A_{i}=\sqrt{2 . \Phi\left(\Omega_{i}\right) \cdot \Delta \Omega}, \quad i=1(1) N$

The applied input of the random road profile shown in Fig. 8 is associated with a roughness of a class $B$, a good road that is modelled by the values in Tab. 6. The NL2D-EWC travels at a constant speed $v_{x}=8 \mathrm{~km} / \mathrm{h}$ and it is supposed that the rear wheel has the same input as the front wheel but delayed by $\delta t=\left(d \cdot \cos \gamma+d_{2}\right) / v_{x}$. The phase angles are distributed 
uniformly and randomly as $\Psi_{i}=2 \pi$.randn(size of $i=1(1) N$ ). The MATLAB function randn produces the same normally distributed pseudorandom numbers each time the program restarts, which is ideal for an optimisation process.

Tab. 6. PSD characteristics of a class B profile

\begin{tabular}{|c|c|c|}
\hline Designation & Value & Unit \\
\hline$N$ & 200 & - \\
\hline$\Omega_{0}$ & 1 & $(\mathrm{rad} / \mathrm{m})$ \\
\hline$\Delta \Omega \equiv\left[\Omega_{\min }, \Omega_{\max }\right]$ & {$[0.05,32.83]$} & $(\mathrm{rad} / \mathrm{m})$ \\
\hline$\Phi_{0}$ & $5.10^{-6}$ & $\left(\mathrm{~m}^{2} /(\mathrm{rad} / \mathrm{m})\right)$ \\
\hline$\omega$ & 2 & - \\
\hline
\end{tabular}

PSD, power spectral density

The road profiles in Fig. 8 are the same, but the phase shift between the continuous black profile, related to the FT, and the dotted magenta profile, linked to the RT, represents the time between the passage of the tires over the same point. These two profiles will be the step inputs $u_{f}$ and $u_{r}$ of the road in the following section.

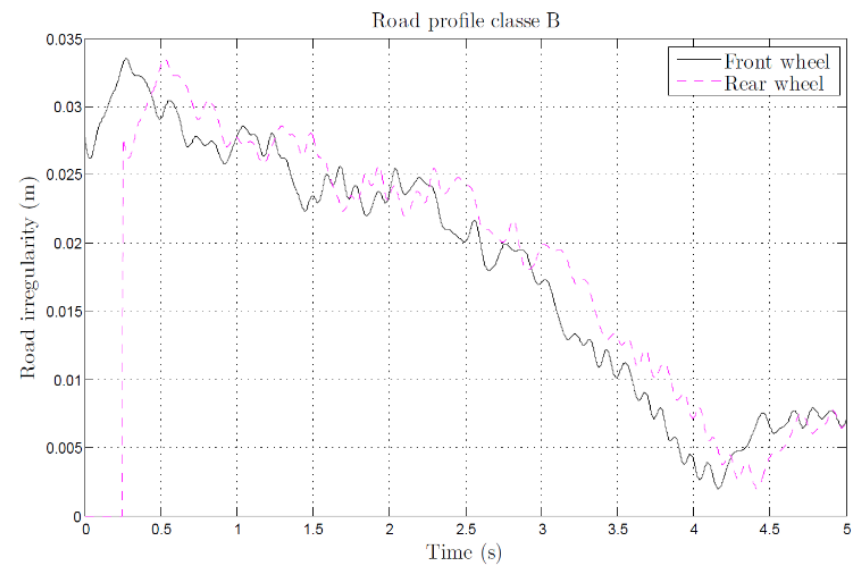

Fig. 8. Excitation for smooth road of Class B

\subsection{Multi-objective optimisation with genetic algorithm}

Considered as meta-heuristics, evolutionary algorithms based on populations of solutions can solve multi-objective problems and generate solutions in the form of fronts. Thus, Deb [6] published in 2001 a book on the use of evolutionary algorithms for multiobjective optimisation, in which his Non-dominated Sorting Genetic Algorithm (NSGA) was upgraded and improved to NSGA-II. During its execution, the genetic algorithm constantly alters a group of solutions to pick individuals from the actual population to be parents. Under some criteria, the chosen parents are served to create a new generation of children, or simply the next individuals. This consecutive creation of generations yields steadily to one or more optimal solutions, depending on the number of objectives ([19]. This work will benefit from the variety of options in the MATLAB Optimisation Toolbox. The genetic algorithm gamultiobj, a variant of NSGA-II (https://www.mathworks.com), will be used to evaluate the effectiveness of the NL2D-EWC model with the options provided in Tab. 7 and the remaining default parameters.
Tab. 7. Problem setting with gamultiobj options

\begin{tabular}{|c|c|}
\hline Designation & Value \\
\hline PopulationSize & 250 \\
\hline CrossoverFraction & 0.8 \\
\hline MaxGenerations & 400 \\
\hline FunctionTolerance & $10^{-4}$ \\
\hline
\end{tabular}

The solutions are shown in the decision-variables space in Fig. 9(a) and the objective space in Fig. 9(b) by using parallelcoords. The solution set is distributed rather irregularly in parallel coordinate plots, which is not a badly-distributed solution set, as uniformly-distributed solutions can have distinct values on different objectives.
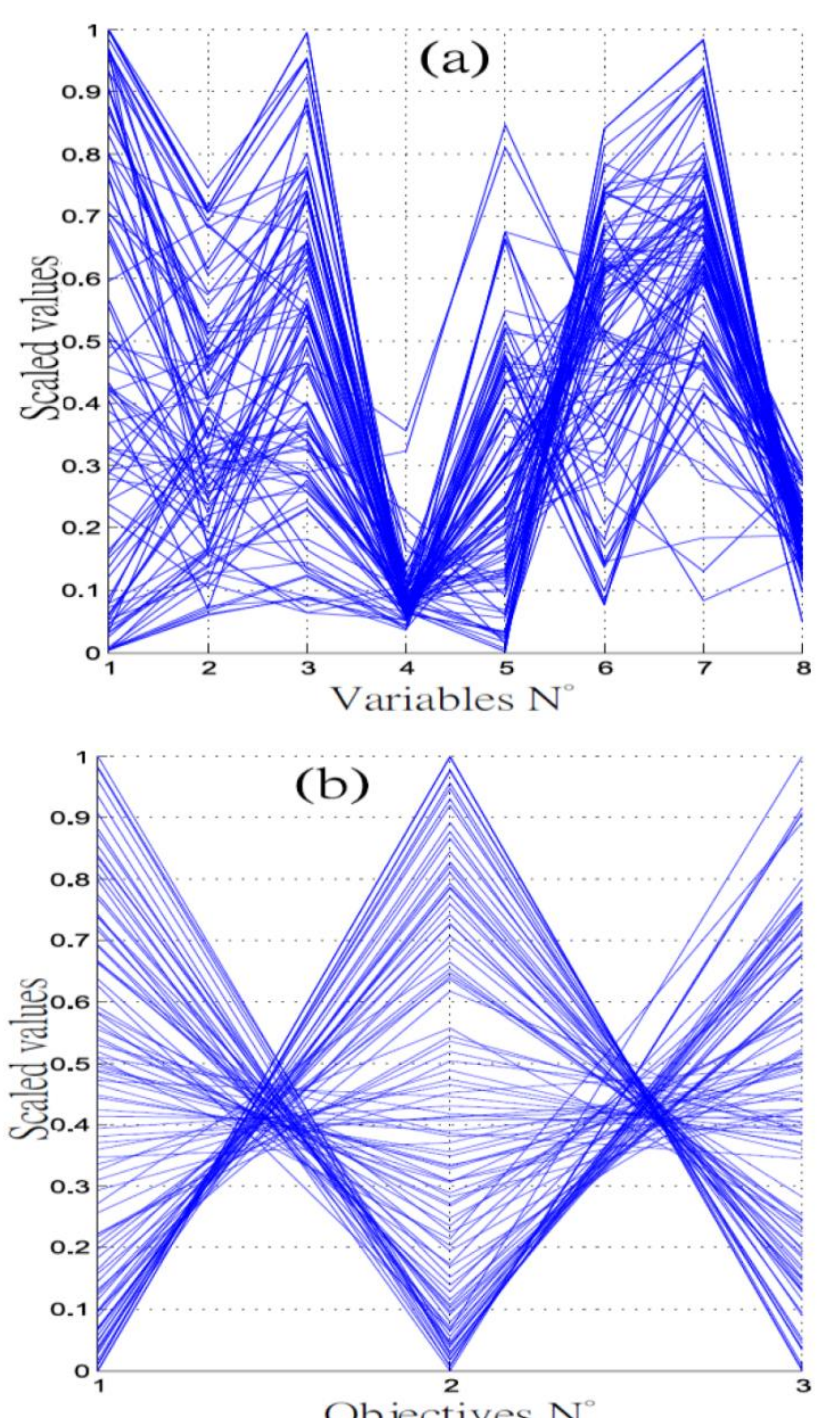

Fig. 9. Parallel Coordinates representation: (a) Sensitivity to the problem parameters, (b) Distribution of solutions

These solutions for the three objectives are illustrated in the 3D Pareto front of Fig. 10. From the variety of solutions, one can recognise those in extremities with the best score for each criterion. It is observed, in the non-dominant solutions of (a), (b) and (c), that the 2D Pareto front of the RMS of vertical acceleration and the Suspension workspace seem to be non-convex and the two 
criteria have almost the same optimal solutions. Otherwise, there

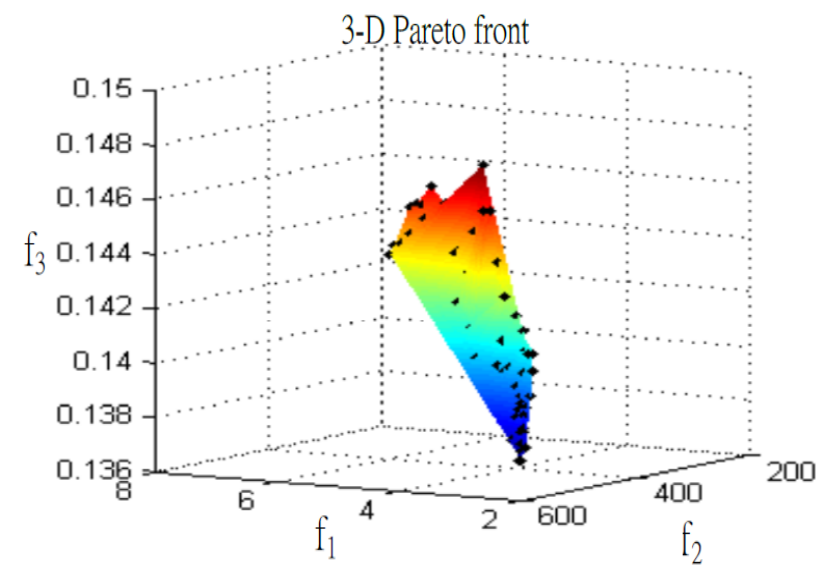

2-D Pareto front (c)

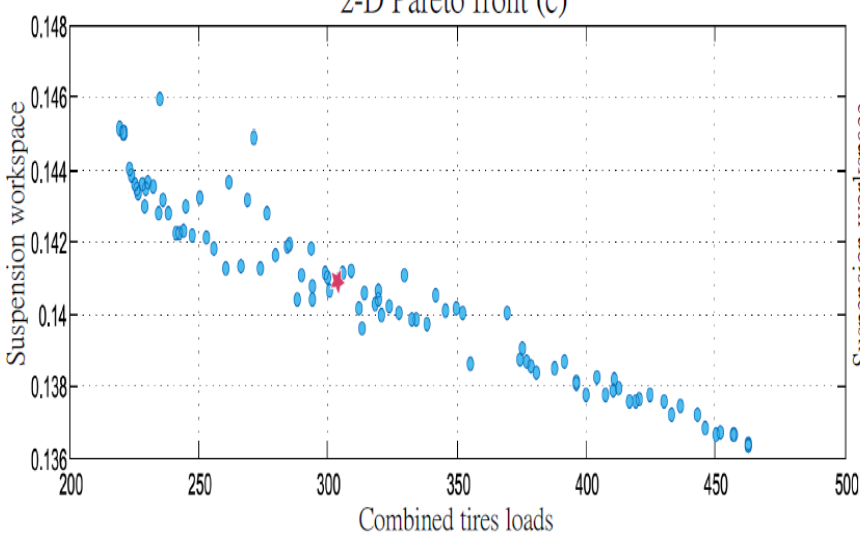

is a clear diversity with the Combined tires' loads.

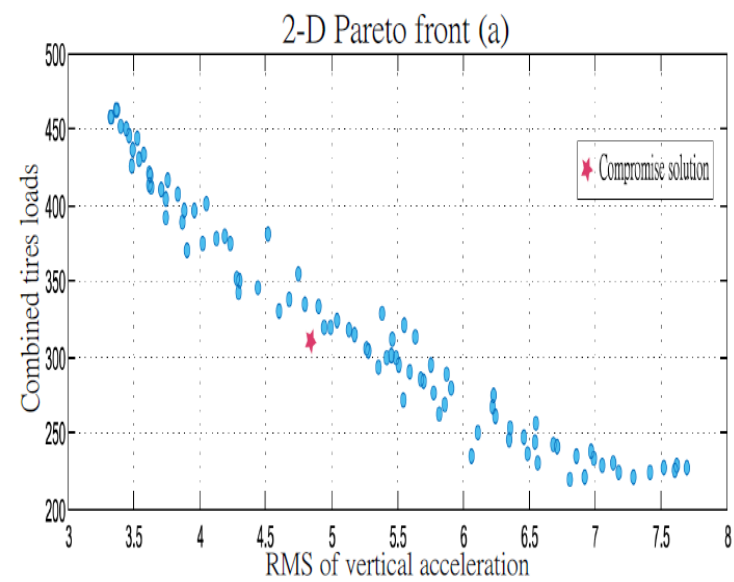

2-D Pareto front (b)

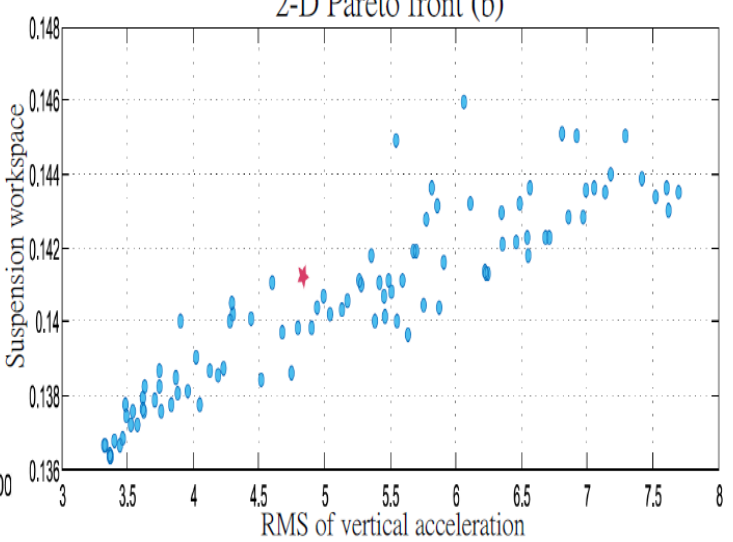

Fig. 10. Surface plot of 3D Pareto front and 2D-Pareto fronts for all combination ( $a, b$, and c). RMS, root-mean-square

The compromised solution, marked by a red star in the figure, can be selected depending on the design preference, but this operation is sometimes difficult. In order to visualise the relation between criteria and facilitate the selection of the compromise point, the decision can be made easier by introducing Level Diagrams (LD) visualisation, which helps to understand the calculated Pareto front and gives correlation between objective and decision space. Reynoso-Meza et al. [25] proposed that each objective $f_{p}(V),(p=1,2$ and 3$)$, is scaled similarly to Eq. (52)

$$
\hat{f}_{p}(V)=\frac{f_{p}-f_{p}^{\min }}{f_{p}^{\max }-f_{p}^{\min }}
$$

For every normalised objective vector $\hat{f}(V)=$ $\left[\hat{f}_{1}(V), \ldots, \hat{f}_{m}(V)\right]$ a $p$-norm ||$\hat{f}(V) \|$ is applied to assess the distance to an ideal and minimal solution at the same time, where

$\left\{\begin{array}{l}\|\hat{f}(V)\|_{1}=\sum_{p=1}^{2} \hat{f}_{p}(V)^{2} \\ || \hat{f}(V) \|_{2}=\sum_{p=2}^{3} \hat{f}_{p}(V)^{2} \\ \|\hat{f}(V)\|_{3}=\hat{f}_{1}(V)^{2}+\hat{f}_{3}(V)^{2}\end{array}\right.$
The LD representation shows a two-dimensional graph for each objective and variable. The arranged sets $\left(f_{p}(V)\right.$, $\left.\|\hat{f}(V)\|_{\mid}\right)$are plotted in each objective and variable sub-graph. Consequently, a prearranged solution will have a similar y-value in all displays. This correspondence makes it possible to compare solutions concurring to the chosen norm according to the general tendencies along the Pareto front. Fig. 11 demonstrates LD plots for each combination, the knee point of which is clearly manifested in all of them. It is noteworthy to observe that the figure is divided into three rows according to the colours. The graphs with red markers are LD for vertical acceleration and combined wheel load, where the minimum value (knee) on the $y$-axis represents the best score for each objective but in relation to the other. The same goes for the yellow and blue rows. The compromise solution shown in the previous figure was selected with help of the LD representations. The results of the optimisation process, with a CPU-time (central processing unit) of $64,679 \mathrm{~s}$, are displayed in Tab. 8. The table compares the scores of the best trade off (corresponding to the compromised solution) with the best candidate in each criterion; considered individually, these scores demonstrate better values. The optimal variables are illustrated as well. 

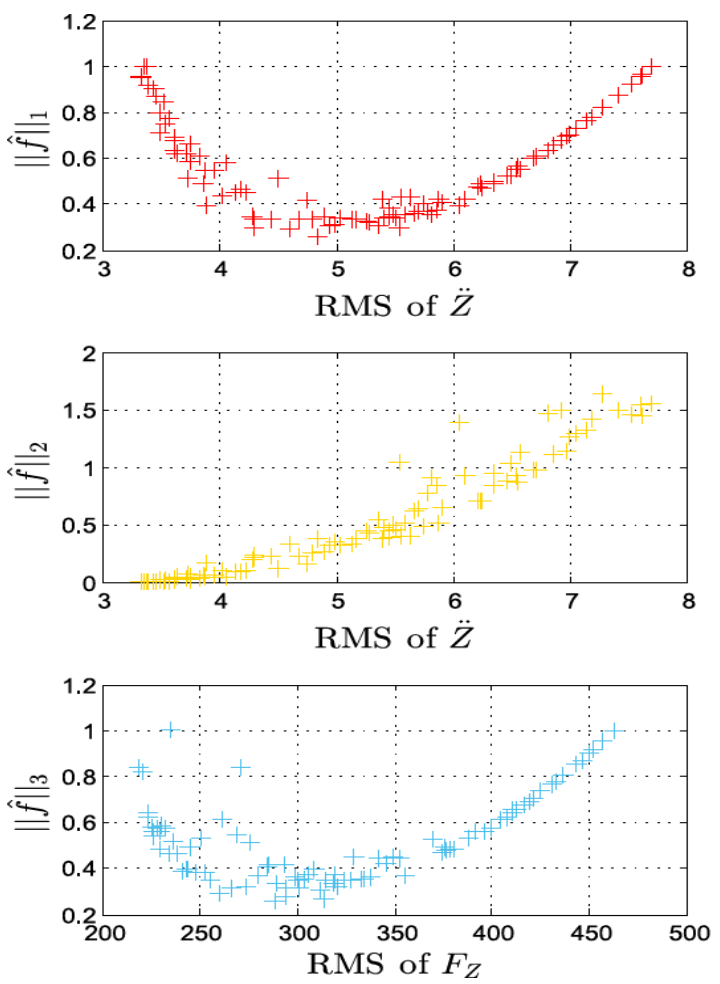
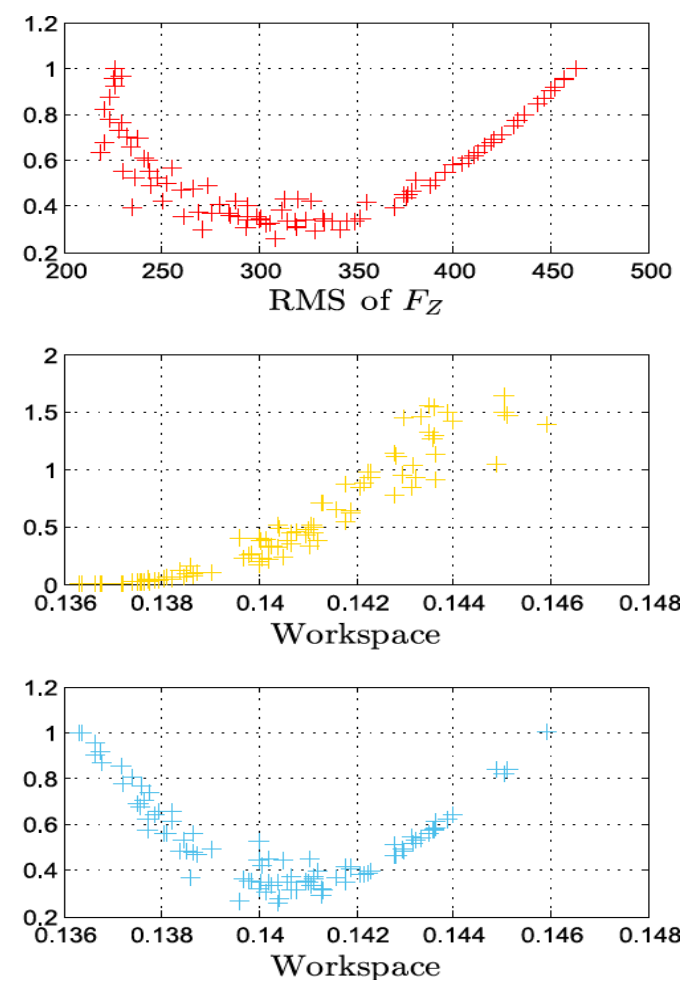

Fig.11. LD representation of Pareto fronts for each bi-objectives. LD, level diagrams; RMS, root-mean-squar

Tab. 8. Best trade off, best candidate in each criterion and optimal variables

\begin{tabular}{|c|c|c|c|}
\hline \multicolumn{2}{|c|}{$\begin{array}{c}\text { Best trade off (compromised } \\
\text { solution) }\end{array}$} & \multicolumn{2}{c|}{$\begin{array}{c}\text { Best candidate in each } \\
\text { criterion }\end{array}$} \\
\hline Designation & Value & Designation & Value \\
\hline$f_{1}$ & 4.8400 & $f_{1}$ & 3.3281 \\
\hline$f_{2}$ & 309.3130 & $f_{2}$ & 219.2769 \\
\hline$f_{3}$ & 0.1412 & $f_{3}$ & 0.1363 \\
\hline \multicolumn{2}{|c|}{ Optimal variables of the compromised solution } \\
\hline Variable & $\begin{array}{c}\text { Optimal } \\
\text { value }\end{array}$ & Variable & $\begin{array}{c}\text { Optimal } \\
\text { value }\end{array}$ \\
\hline$m_{S}$ & $64.8411 \mathrm{~kg}$ & $K_{S}$ & $14,477 \mathrm{~N} / \mathrm{m}$ \\
\hline$m_{C}$ & $1.3581 \mathrm{~kg}$ & $N_{S}$ & $\begin{array}{c}1,932.6 \\
\mathrm{~N} . \mathrm{s} / \mathrm{m}\end{array}$ \\
\hline$m_{F T}$ & $3.4178 \mathrm{~kg}$ & $c_{z f}$ & $96,210 \mathrm{~N} / \mathrm{m}$ \\
\hline$m_{R T}$ & $5.5654 \mathrm{~kg}$ & $c_{z r}$ & $91,481 \mathrm{~N} / \mathrm{m}$ \\
\hline
\end{tabular}

slight difference in the values of the best compromise and the best candidate in each criterion, especially for the combined tires' load. This is due to the non-dominant role of the mass $\mathrm{ms}$ in the motion of the FT and the favouring of the coefficient $\kappa_{f}\left(\kappa_{f}=\right.$ 0.6 and $\kappa_{r}=0.4$ ) in the formula for this objective (f2) in Eq. (49). Optimisation with user's mass has shown that for high Sprung mass or high user's mass, the RMS of vertical acceleration and the suspension workspace will be lower. However, for the load of the tires it is the opposite.

The simulations presented previously in the optimisation process did not take into account the mass of the user. To increase the results, a new simulation was run by adding a fixed average additional mass $(80 \mathrm{~kg})$ to the variable mass of the Sprung mass. Tab. 9 contains a comparison between the values without and with the user's mass.

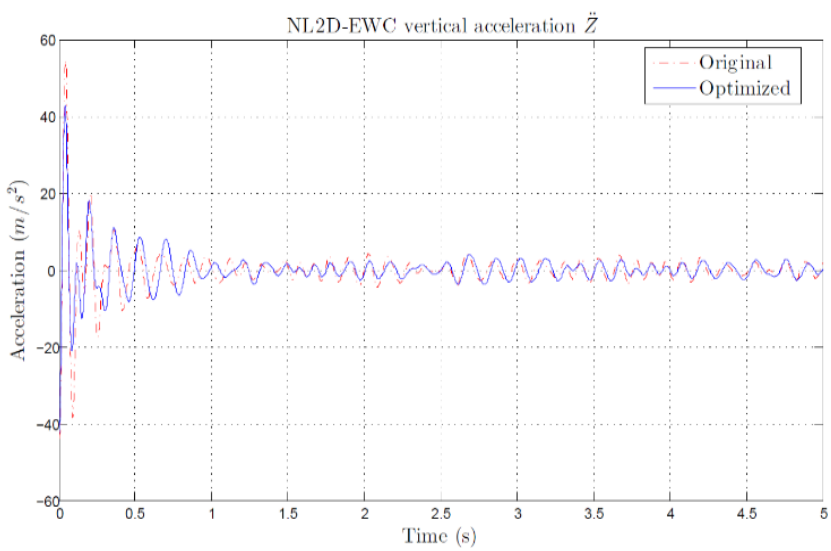

Fig.12. Vertical acceleration of the sprung mass: Optimised vs. origin. NL2D-EWC, non-linear two-dimensional electric wheelchair 

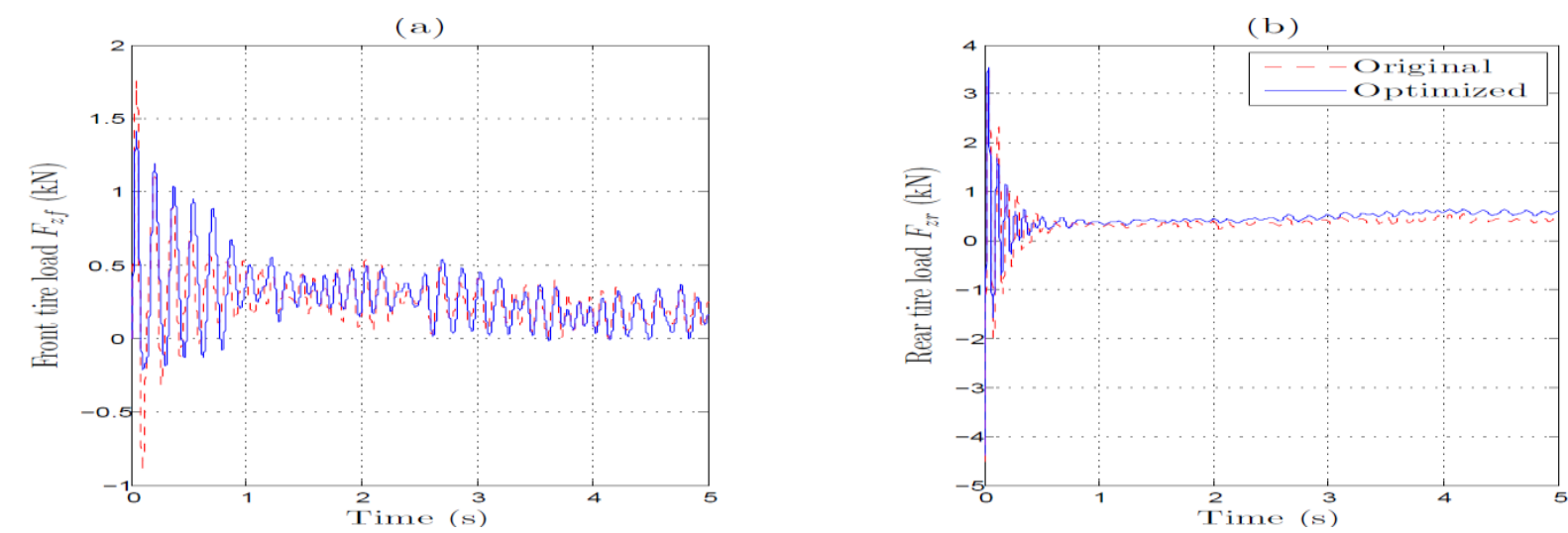

Fig.13. Wheel's load: (a) FT load: Optimised vs. origin, (b) RT load: Optimised vs. origin. FT, front tire; RT, rear tire

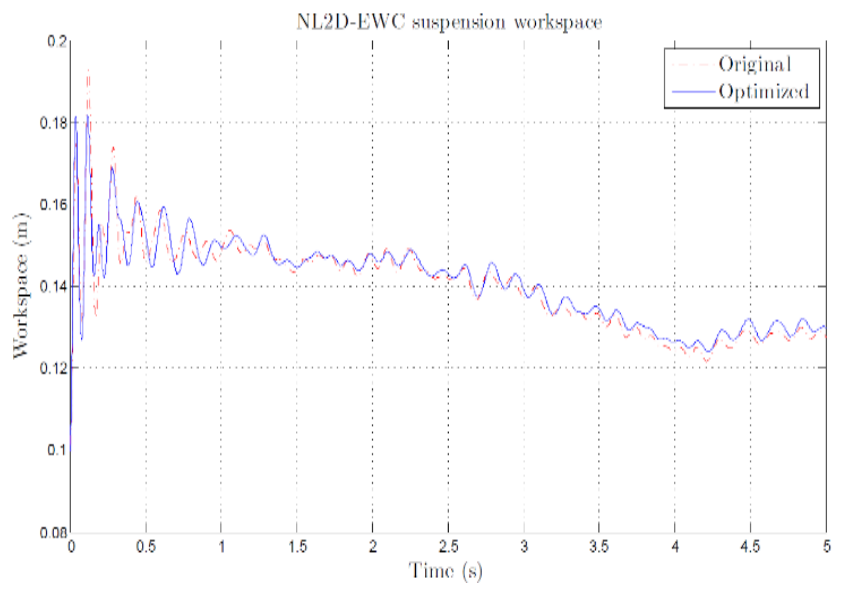

Fig.14. Suspension workspace: Optimised vs. origin. NL2D-EWC, non-linear two-dimensional electric wheelchair

Tab. 9. Best trade off, best candidate in each criterion of the optimisation without and with the user's mass

\begin{tabular}{|c|c|c|c|c|}
\cline { 2 - 5 } \multicolumn{1}{c|}{} & \multicolumn{2}{c|}{$\begin{array}{c}\text { Optimisation without } \\
\text { the user's mass }\end{array}$} & \multicolumn{2}{c|}{$\begin{array}{c}\text { Optimisation with the } \\
\text { user's mass }\end{array}$} \\
\cline { 2 - 5 } & $\begin{array}{c}\text { Best trade } \\
\text { off }\end{array}$ & $\begin{array}{c}\text { Best } \\
\text { candidate }\end{array}$ & $\begin{array}{c}\text { Best } \\
\text { trade off }\end{array}$ & $\begin{array}{c}\text { Best } \\
\text { candidat } \\
\text { end }\end{array}$ \\
\hline Objective & Value & Value & Value & Value \\
\hline$f_{1}$ & 4.8400 & 3.3281 & 2.1924 & 1.9229 \\
\hline$f_{2}$ & 309.3130 & 219.2769 & 792.9069 & 569.6984 \\
\hline$f_{3}$ & 0.1412 & 0.1363 & 0.1312 & 0.1280 \\
\hline
\end{tabular}

\section{CONCLUSION}

This paper presents a description of Jourdain's principle through its application in modelling an electric wheelchair. Therefore, the equations of motion were formulated on this interpretation to facilitate their comprehension. The approach provided advantages over other classical formulations. The concept of generalised coordinates and velocities was used to embed the constraints and derive first-order differential equations. An appropriate choice of velocities conducts to decoupled second derivatives. The method also suggested that the virtual power of constraint forces vanishes from the beginning, which was another advantage. The straightforward implementation of the principle was noteworthy and the reduced number of symbolic equations characterised the efficiency of the calculations.

Despite the slight dissimilarities, the comparison between the responses of the two-dimensional non-linear model and the three-dimensional one built into the simulation software showed that a precise setting in the multi-body modelling of mechanical systems could offer great results in a short time with low processing capacity requirements.

The improved ride comfort, road holding and working space of the suspension made it possible to test the effectiveness of the model. The objective cost functions for these criteria have been defined and inserted into a MATLAB multi-objective genetic algorithm. The scaling of the variables allowed a balanced scanning in their intervals. Acceptable solutions were captured on the Pareto fronts, but the non-convexity between ride comfort and the suspension workspace was noticeable, making the latter unprofitable. While the FT load was initially preferred over the rear load, the final decision of the compromise solution between all criteria was estimated by using the LD visualisation. It has been noticed that the generation of a random road profile by the PSD considerably increased the processing time.

\section{REFERENCES}

1. Ahmad S, Tokhi M, Toha S. Genetic Algorithm Optimisation for Fuzzy Control of Wheelchair Lifting and Balancing. Uksim European Symposium On Computer Modeling And Simulation. 2009.

2. Anandan $A$, Kandavel $A$. Investigation and performance comparison of ride comfort on the created human vehicle road integrated model adopting genetic algorithm optimized proportional integral derivative control technique. Proceedings Of The Institution Of Mechanical Engineers Part K: Journal Of Multi-Body Dynamics. 2020; 234(2): 288-305.

3. Balkwill J. Performance vehicle dynamics: Engineering and Applications. Elsevier. 2018.

4. Chen S, Shi T, Wang D, Chen J. Multi-objective optimization of the vehicle ride comfort based on Kriging approximate model and NSGA-II. Journal Of Mechanical Science And Technology. 2015; 29(3):1007-1018.

5. Dad K, Khan M, Jie W, Lee M. A low cost genetic algorithm based control scheme for wheelchair control in hospital environment. Proceedings Of International Conference On Artificial Life And Robotics. 2016; 21:212-216.

6. Deb K. Multi-objective optimization using evolutionary algorithms. John Wiley \& Sons, Ltd. 2001.

7. Dodds $\mathrm{C}$, Robson J. The description of road surface roughness. Journal Of Sound And Vibration. 1973; 31(2):175-183. 
8. Fossati G, Miguel L, Casas W. Multi-objective optimization of the suspension system parameters of a full vehicle model. Optimization And Engineering. 2018; 20(1):151-177.

9. Garcia de Jalon J, Bayo E. Kinematic and dynamic simulation of multibody systems. Springer. 1984.

10. Gill P, Murray W, Wright M. Practical optimization. SIAM. 2019.

11. Hahn H. Rigid body dynamics of mechanisms. Springer. 2003.

12. https://www.karmanhealthcare.com/product/xo-202/ [online cit.: 2020.06.16].

13. https://www.mathworks.com/help/gads/gamultiobj-algorithm.html. [online cit:: 2021.01.29].

14. Hurel J, Mandow A, Garcia-Cerezo A. Nonlinear two-dimensional modeling of a McPherson suspension for kinematics and dynamics simulation. 12 ${ }^{\text {Th }}$ IEEE International Workshop On Advanced Motion Control (AMC). 2012

15. Jazar R. Vehicle dynamics - theory and application (3 ${ }^{\text {rd }}$ ed.). Springer. 2017

16. Jourdain P. Note on an Analogue of Gauss Principle of Least Constraint. The Quarterly Journal of Pure and Applied Mathematics. 1909; 40:153-157.

17. Kane T. Dynamics of Nonholonomic Systems. Journal Of Applied Mechanics. 1961; 28(4):574-578.

18. Kane T, Levinson D. The Use of Kane's Dynamical Equations in Robotics. The International Journal Of Robotics Research. 1983; 2(3):3-21.

19. Messac A. Optimization in practice with MATLAB for engineering students and professionals. Cambridge University Press. 2015.

20. Nariman-Zadeh N, Salehpour M, Jamali A, Haghgoo E. Pareto optimization of a five-degree of freedom vehicle vibration model using a multi-objective uniform-diversity genetic algorithm (MUGA):Engineering Applications Of Artificial Intelligence. 2010; 23(4):543-551.

21. Pacejka $H$, Besselink I. Tire and vehicle dynamics. Elsevier/BH. 2012.

22. Papastavridis J. On Jourdain's principle. International Journal Of Engineering Science. 1992; 30(2):135-140.

23. Paulter N, Larson D, Blair J. The IEEE Standard on Transitions. Pulses. and Related Waveforms. Std-181-2003. IEEE Transactions On Instrumentation And Measurement. 2004; 53(4):1209-1217.

24. Piedboeuf J. Kane's equations or Jourdain's principle?. Proceedings Of $36^{\text {Th }}$ Midwest Symposium On Circuits And Systems. 1993.
25. Reynoso-Meza G, Blasco X, Sanchis J, Herrero J. Comparison of design concepts in multi-criteria decision-making using level diagrams. Information Sciences. 2003; 221:124-141.

26. Rill G. Road vehicle dynamics. CRC Press. 2012.

27. Roberson R, Schwertassek R. Dynamics of Multibody Systems. Springer Berlin Heidelberg. 1988.

28. Sankardoss V, Geethanjali P. Parameter estimation and speed control of a PMDC motor used in wheelchair. Energy Procedia. 2017; 117:345-352.

29. Seifi A, Hassannejad R, Hamed M. Use of nonlinear asymmetrical shock absorbers in multi-objective optimization of the suspension system in a variety of road excitations. Proceedings Of The Institution Of Mechanical Engineers. Part K: Journal Of Multi-Body Dynamics. 2016; 231(2):372-387.

30. Shabana A. Computational dynamics. John Wiley \& Sons. 2010

31. Shirahatti A, Prasad P, Panzade P, Kulkarni M. Optimal design of passenger car suspension for ride and road holding. Journal Of The Brazilian Society Of Mechanical Sciences And Engineering. 2008; 30(1):66-76.

32. Sinha B. Influence of road unevenness on road holding and ride comfort. Stockholm: Department of Machine element deesign. Royal Institute of Technology. 1973

33. Van der Sande T, Besselink I, Nijmeijer H. Rule-based control of a semi-active suspension for minimal sprung mass acceleration: design and measurement. Vehicle System Dynamics. 2016; 54(3):281-300.

34. Vingback J, Jeppsson P, van Deventer J. Evaluating Ride Comfort for Wheelchair Passengers Utilizing a Motionbase Simulator. $16^{\text {Th }}$ International Conference On Advanced Vehicle Technologies; 11 $11^{\text {Th }}$ International Conference On Design Education; $7^{\text {Th }}$ Frontiers In Biomedical Devices. 2014; 3.

35. Wang S, Zhao L, Hu Y, Yang F. Vibration Characteristics Analysis of Convalescent-Wheelchair Robots Equipped with Dynamic Absorbers. Shock And Vibration. 2018; 1-16.

Mohamed Belhorma: (D) https://orcid.org/0000-0001-7063-4170

Aboubakar S. Bouchikhi: (D) https://orcid.org/0000-0002-3389-9177 